\title{
Theoretical and methodological approaches to apparent movement of short-range process and pattern perception
}

\author{
JIH JE CHANG \\ AT\&T Bell Laboratories, Murray Hill, New Jersey
}

\begin{abstract}
A variable raster and vector display processor interfaced with a PDP-11/23 computer is briefly described. Several experiments using random-dot cinematograms (RDCs) (raster display) and line segments (vector display) are discussed. The experiments on cooperative processes of apparent movement of RDCs and spatial-frequency-filtered RDCs showed that the average cooperative neighborhood of neural interaction is about $15 \mathrm{~min}$ arc of visual angle. In pattern perception, based on Julesz's texton theory (1981) and Shaw's attention model (1980), this study showed that the aperture of attention in serial and parallel processing is a function of feature gradient, set size, and eccentricity.
\end{abstract}

Since the early 1970s, vision research in AT\&T Bell Laboratories has been centered on the early visual processes of stereoscopic and movement perception, using random-dot stereograms (RDSs) and random-dot cinematograms (RDCs), and on texture and feature discrimination. The computer that carried out these experiments was a PDP-11/23 interfaced with a variable raster and vector display processor designed and built by Walter Kropfl. In this paper, I describe several experiments on apparent movement of the short-range process using the raster display mode and a study involving attentional effects in feature discrimination using the vector display mode.

\section{THE VARIABLE RASTER AND VECTOR DISPLAY PROCESSOR (VRVDP)}

The VRVDP was interfaced with two computers, a PDP-11 computer using a DR11-B interface and a GA1830 computer with a built-in DMA (direct memory access) channel for fast transmission. The stimulus was displayed on a Hewlett-Packard electrostatic graphic display model 1311A. The scope has a short settling time of less than $200 \mathrm{nsec}$ for small steps and less than $1 \mu \mathrm{sec}$ for on-screen movement. DATEL HI series 10-bit analogto-digital converters with a very short settling time (25 nsec) were used to drive the $x$ and $y$ axes of the scope. In the raster mode, VRVDP decodes a sequence of 16bit words input as either instructions or data-intensification information. The commands dictate what rectangular area should be displayed and how it should be displayed. The options include the starting $x, y$ coordinates, the lengths of the rectangular area, the spacing between dots, the in-

I wish to thank Walter Kropfl for providing me with information on the display system. I thank Bela Julesz for his helpful comments and support. I am deeply grateful to Bart Farrell for his invaluable suggestions and his help in editing this paper.

The author's mailing address is: AT\&T Bell Laboratories, Murray Hill, NJ 07974. tensity of the dots, and the direction of scanning in the $x$ and $y$ planes. To display a random-dot image, it takes $1 \mu \mathrm{sec}$ to place a dot in incrementing or decrementing positions, of which $0.4 \mu \mathrm{sec}$ is for settling time before intensification and $0.6 \mu \mathrm{sec}$ is for the intensification period. For initialization, it takes $1.5 \mu \mathrm{sec}$ to intensify the first point or a jump. The vector display system uses a hardware implementation of Bresenham's (1965) algorithm. The commands determine the size and direction of a vector. The addition of a hidden vector bit provides the flexibility of connecting a complex pattern by hidden vectors.

\section{EXPERIMENTS}

The research is described in two parts. In Part 1, I summarize two experiments on the cooperative processes of apparent movement of RDCs (Chang \& Julesz, 1984, 1985). In Part 2, I describe an experiment based on Julesz's (1981) texton theory and Shaw's (1980) attention model.

\section{Apparent Movement of the \\ Short-Range Process}

The term "short-range process" (SRP) was introduced by Braddick (1974) to describe a kind of apparent movement that is distinctly different from classical apparent movement, which is mediated by the long-range process (LRP). The best example for SRP is the phenomena associated with RDCs. Computer-generated random-dot images date back to 1960 when Julesz used RDSs to investigate stereoscopic vision (Julesz, 1960) and Julesz and Payne (1968) compared moving RDSs and RDCs. Because RDSs and RDCs are similar in that both involve matching processes, the many theories and computational models of stereo vision developed over the past 25 years (Julesz, 1962, 1971; Julesz \& Chang 1976; Marr \& Poggio, 1976, 1979; Nelson, 1975; Sperling, 1970) can be generalized to the perception of short-range movement in RDCs. 
Unlike conventional stereoscopic stimuli, RDSs are void of monocular cues, such as edges or contours. For a person to see an area of a RDS in uniform depth, dots in each eye's view must be shifted horizontally in opposite directions to create crossed or uncrossed disparities (Julesz, 1960, 1964, 1971). Since each eye sees numerous black and white dots of identical appearance, the question arises as to how the visual system disambiguates all the possible pairing of dots between the two eyes' views. Julesz claimed that the matching problem in stereopsis and movement is solved by a global and cooperative process. Previous studies bearing on global processing have examined maximum disparity or maximum displacement $\left(D_{\max }\right)$, which is defined as the upper limit for discriminating crossed or uncrossed depth planes in stereopsis or the direction of motion in apparent movement. These studies have shown that $D_{\max }$ for both stereopsis and motion increases with the square root of the stimulus area (Chang \& Julesz, 1983; Lappin \& Bell, 1976; Tyler \& Julesz, 1980). This result supports Julesz's hypothesis of global processing. For cooperative processing, Julesz and Chang (1976) used ambiguous RDSs to study cooperative processes analogous to the multistable state phenomena in physics. In an ambiguous RDS, two depth planes of equal strength (100\% of dots match in both directions) coexist. For an unbiased observer, the probability of perceiving the depth plane in front or behind is equal. At any single moment, however, only one percept can be seen. Julesz and Chang found that if $8 \%$ of the dots were unmatched in, say, the front plane, then the otherwise ambiguous percept became unambiguous, and the target plane was always perceived to be behind the fixation point and never in front. This cooperative phenomenon in stereopsis has led Julesz and Chang to explore it further, but in the area of apparent movement instead of stereopsis. The reason for doing so is because the experimental methodology is more suitable for studying movement than for depth perception. Two studies on cooperative phenomena of apparent movement of the SRP have been published (Chang \& Julesz, 1984, 1985). In both studies RDCs were generated by Kropfl's variable raster and vector display processor (VRVDP).

Experiment 1: Ambiguous random-dot cinematograms. The stimulus was composed of alternating horizontal stripes of ambiguous and unambiguous random dots (Figure 1). Like the RDSs, the ambiguous RDCs were perceived to move uniformly either to the left or to the right with equal probability. An ambiguous RDC is composed of repetitive columns of A and B patterns. As illustrated in Figure 2, if the second image is shifted by the width of $A$, where the widths of $A$ and $B$ are equal, then dots in the two patterns can be matched either to the right or to the left. This ambiguous percept can be changed, however, by introducing bias in one of the directions. A bias is created by complementing the dot in the first image and its corresponding dot in the second image only in the biased direction, thus making the pair in the
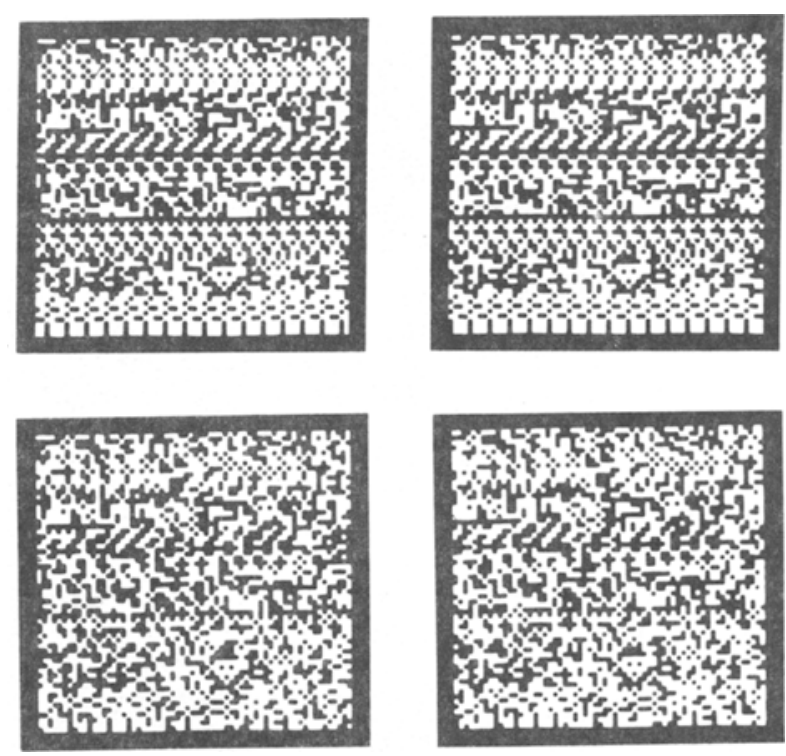

Figure 1. Random-dot cinematograms composed of eight alternating ambiguous and unambiguous stripes with two-dot displacement. Top pair has no bias in the ambiguous areas. Bottom pair has $25 \%$ of bias in the ambiguous areas. From "Cooperative phenomena in apparent movement perception of random-dot cinematograms" by J. J. Chang and B. Julese, 1984, Vision Research, 24, 1783. Reprinted with permission.

opposite direction unmatched. The RDC was stored in a linear array and was displayed by the VRVDP at a $40-\mathrm{Hz}$ frame rate for $120 \mathrm{msec}$ at $60 \mathrm{msec}$ per image. Preliminary results showed that if the number of bias dots inserted in the ambiguous $\mathrm{RDC}$ reached $6 \%-8 \%$ of the total, then the direction of movement would always be perceived in the biased direction. In the alternating ambiguous and unambiguous stripes, the direction of movement in the unambiguous areas was always opposite to the biased direction in the ambiguous areas. The objective of using juxtaposed areas moving in opposite directions was to observe if any nonlinear interactions between polarization would result in the ambiguous areas being pulled by the forces of the unambiguous areas. In fact, pulling of the ambiguous areas by the unambiguous areas was found to depend on the amount of bias inserted in the ambiguous areas. Moreover, the percent of bias needed to be free of the pulling is a function of the height of the stripe. A cancellation paradigm, used to compute the index of cooperative strength (CS), indicated that the average cooperative neighborhood or the area of nonlinear interaction between adjacent areas is about 15 min arc of visual angle. (For a detailed description see Chang and Julesz, 1984.)

Experiment 2: Spatial-frequency-bandpassed random-dot cinematograms. The second study was intended to investigate two controversial hypotheses concerning cooperative versus noncooperative processes in stereopsis. The hypotheses are extended to apparent movement of the SRP. Marr and Poggio (1979) formulated a noncooperative model of stereopsis based on Logan's zero- 


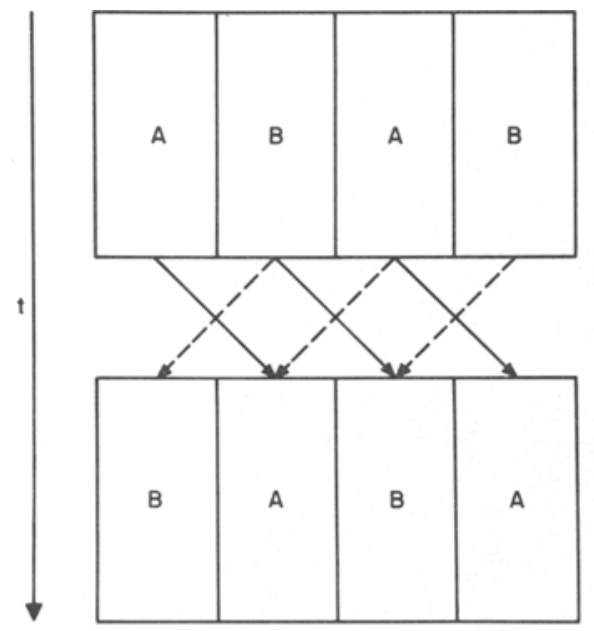

AMBIGUOUS RANDOM - DOT CINEMATOGRAMS $\longrightarrow$ PERCEIVED MOVEMENT 1 $--\rightarrow$ PERCEIVED MOVEMENT 2

Figure 2. Ilustrations of vertical periodic patterns (ABAB ...) in ambiguous random-dot cinematograms. Corresponding dots between the two frames are correlated $100 \%$ in both directions as shown by solid and dotted arrows. From "Cooperative phenomena in apparent movement perception of random-dot cinematograms" by J. J. Chang and B. Julesz, 1984, Vision Research, 24, 1782. Reprinted with permission.

crossing theorem (1977) for eliminating false matches. They stated that if disparity is one-half the width of the receptive field, then the probability of a correct match is $95 \%$. They also claimed that the matching process is not necessarily cooperative, because disparity information can be processed by units tuned to corresponding receptive-field size; that is, large units process lowfrequency images and small units process high-frequency images (Wilson \& Bergen, 1979). Generalizing Marr and Poggio's model to apparent movement of the SRP, maximum displacement should be linearly related to the spatial frequency of the image. The cooperative model (Julesz, 1971), on the other hand, does not call for a relationship between spatial frequency and $D_{\max }$, and we can assume that maximum displacement is invariant over spatial frequency. Filtered random-dot images with oneoctave bandwidth were generated on a Honeywell DSP8/70 computer by a Fourier analysis program (Cooley \& Tukey, 1965). The spatial frequency of the eight bandpassed images ranged from 6-12 cycles per image (cpi) to 24-48 cpi. The original 256 gray-level image was quantized into eight gray levels for real-time display. The quantized image was decomposed into three black-and-white subimages. The intensity control of the VRVDP was set at various levels for each of the three subfields to obtain the desired combination of contrasts. Each dot in the subfield took one bit of memory. Corresponding bits in the three subfields were superimposed to obtain the intensity level of a single random dot. Because of limited core memory, only one filtered image was generated for each spatial frequency; however, each image could be made to appear as eight different images by combinations of scanning in the up-down and/or left-right directions. Four filtered stimuli with eight intensity levels are shown in Figure 3 (from Chang \& Julesz, 1985). The three $96 \times 96$ dot subimages were presented sequentially with an integration time of $40 \mathrm{msec}(25 \mathrm{~Hz})$, and the stimulus duration on each trial was $160 \mathrm{msec}$.

Data for two subjects (Figure 4; see Chang \& Julesz, 1985 , for detailed discussion), with center frequencies ranging from 1-10 cpd, shows that maximum displacement $\left(D_{\max }\right)$ decreases linearly with an increase in spatial frequency. This supports Marr and Poggio's (1979) noncooperative model. At spatial frequency of $4 \mathrm{cpd}$ and above, however, $D_{\max }$ is independent of spatial frequencies and is nearly constant. This agreement of $4 \mathrm{cpd}$ (15 min arc) between the two studies reinforced our claim that the average cooperative area of neural processing in apparent movement of the SRP is around $15 \mathrm{~min}$ arc. This $15 \mathrm{~min}$ arc cooperative area might also be related to the average size of the cortical hypercolumn, which is $0.25^{\circ}$ in the macaque monkey (Hubel \& Wiesel, 1974).

\section{Preattentive and Attentive Processes: \\ Primitive Features and Texton Theory}

The distinction between the attentive and preattentive processes of texture patterns has long been of interest to psychologists. Recent theories have framed the distinction in terms of the processing of primitive features, such as line segments, orientation, color, luminance, and movement. Since the discovery of feature detectors in the cor-
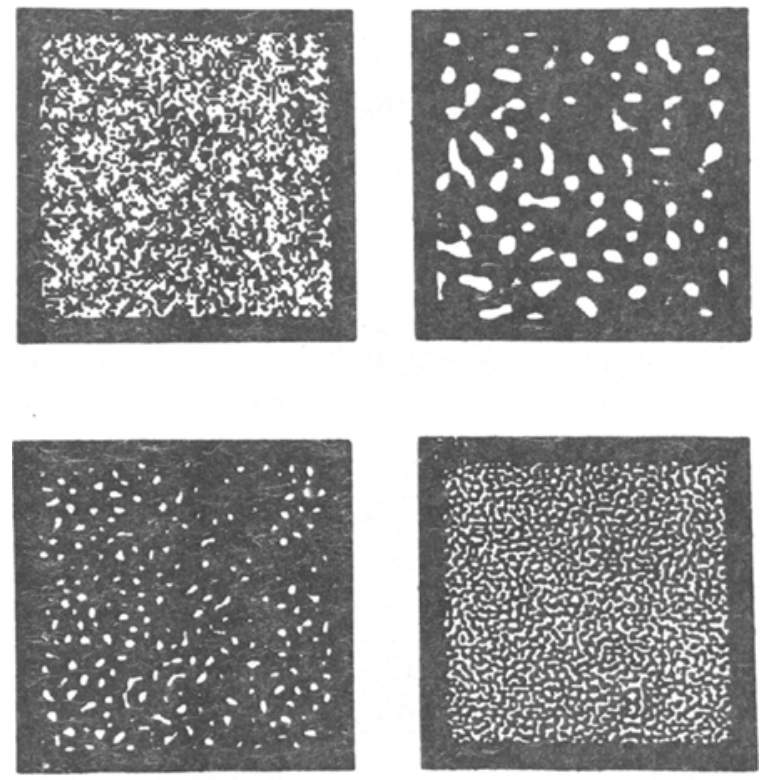

Figure 3. Clockwise from upper left: the unfiltered $96 \times 96$ randomdot image; bandpass (6-12 cycles per image [cpi] width); bandpass (24-48 cpi width); handpass (12-24 cpi width). From "Cooperative and non-cooperative processes of apparent movement of randomdot cinematograms " by J. J. Chang and B. Julesz, 1985, Spatial Vision, 1(1), 42. Reprinted with permission. 

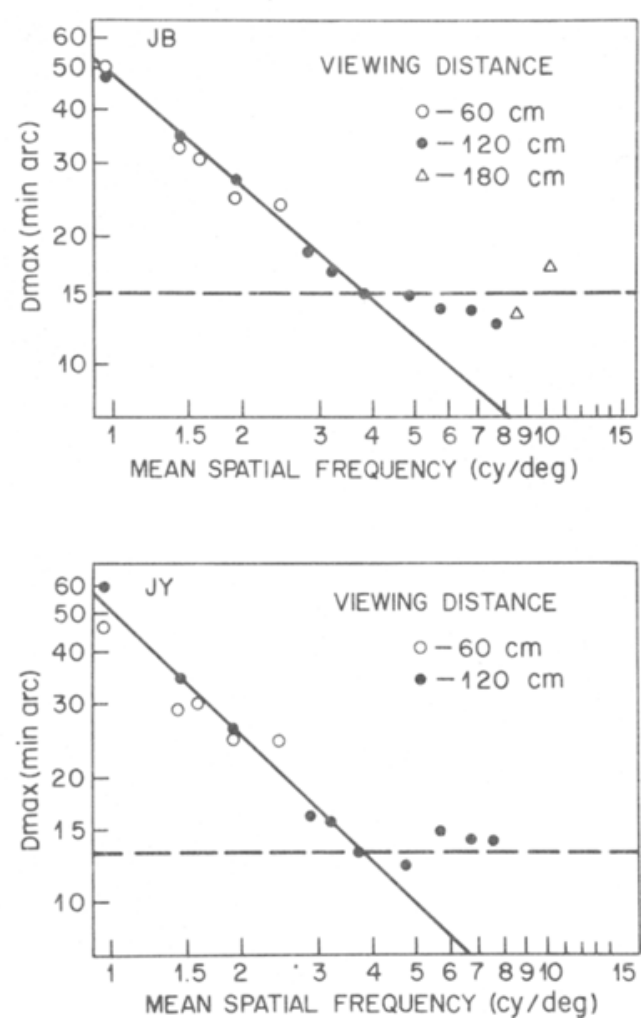

Figure 4. Maximum displacement $\left(D_{\max }\right)$ as a function of mean spatial frequencies of the bandpassed images in cycles per degree. Solid line represents Marr and Poggio's (1979) prediction of the noncooperative model. For the cooperative model, we simply fitted the data with a horizontal line to show that $D_{\max }$ is independent of spatial frequencies. From "Cooperative and non-cooperative processes of apparent movement of random-dot cinematograms" by J. J. Chang and B. Julesz, 1985, Spatial Vision, 1(1), 43. Reprinted with permission.

tex of cats and monkeys (Hubel \& Wiesel, 1959, 1968), explanations of many perceptual phenomena have benefitted from neurological evidence. Specifically, these primitive features (or differences between them) are detected automatically, preattentively, and in parallel, but detecting combination of features requires attention in the form of serial search (Julesz, 1981; Treisman \& Gelade, 1980). For example, serial attentive processing is required to detect one $\mathrm{T}$ among a background of Ls, because the same two lines in different combinations can make either a $\mathrm{T}$ or an L. Julesz claims, however, that when the same two lines are coupled to form a + rather than a $T$, then detecting the + among Ls is parallel and preattentive because the crossing of two line segments, which is found in + but not in $\mathrm{L}$, is a primitive feature.

Bergen and Julesz (1983a) studied the effects of attention on feature discrimination using such features as $T$, $\mathrm{L}$, and + made up of equal line lengths and orientations. Using briefly flashed $(100-\mathrm{msec})$ patterns. They compared the accuracy of detecting one target $\mathrm{L}$ among a variable number of distractor $+\mathrm{s}$ with that of detecting one target $\mathrm{L}$ among Ts. For an $\mathrm{L}$ embedded in a field of $+\mathrm{s}$, they found that as the number of $+\mathrm{s}$ increased, performance dropped only slightly, from $100 \%$ correct to over $90 \%$ correct. However, for an $\mathrm{L}$ in a field of Ts, performance decreased steadily from $90 \%$ to below $65 \%$. When one target among 35 distractors was tested as a function of inspection time (or stimulus onset asynchrony-SOA), Bergen and Julesz showed that for an $L$ in a field of $+s$, performance was perfect at $160 \mathrm{msec}$, but for an $\mathrm{L}$ in a field of Ts, it reached an asymptote of $62 \%$ correct at about 300 msec. These results provided evidence for the existence of two visual systems: a preattentive system that can discriminate simple feature differences rapidly and in parallel, and an attentive system associated with serial processing. Julesz (1981) showed that the preattentively discriminable features are local and called these features textons. Textons are elongated blobs of various width, length, color, orientation, terminators, and crossings. Bergen and Julesz (1983a) suggested that since the detection of an $\mathrm{L}$ among $+\mathrm{s}$ does not depend on the number of target locations (i.e., set size), it is a parallel process, whereas the detection of an $\mathrm{L}$ among $\mathrm{Ts}$ does and is, therefore, a serial process. If we assume that serial processing is done by sequentially inspecting a subfield of the entire display, then performance should depend on the size and the number of subfields attended within the time limit (SOA). The study described below explores how performance is affected by feature gradient ( $T, L$, and + ), set size, and eccentricity based on Julesz's texton theory and Shaw's attention model $(1980,1982)$. Julesz's texton theory deals specifically with the effects on both preattentive and attentive processes of the differences between texture gradients. Shaw's attention model, however, centers on the set-size effect and defines an upper bound for performance decrement, which is due only to attentional factors.

Shaw (1980) formulated a theory of division of attention. Like signal detection theory, it postulated two stages in information processing: an internal coding stage where sensory coding, independent at each source, is represented by a random variable; and a decision-making stage, which is affected by the choice of criterion. Central to Shaw's theory is a new method of examining attentional factors with respect to the effect of set size (i.e., the number of target locations). The set-size effect is assumed to be influenced by both attentional and decision-making factors. Using detection and location judgment experiments with a variable set size, Shaw was able to separate attentional factors from the decision-making process. Shaw proved that when the number of information sources increased from 2 to $N$ locations, the upper bound for the performance decrement due to decision making is $P_{2}-P_{2}^{N-1}$, where $P_{2}$ represents the probability of correct localization in a two-location search. Any decrement that exceeds this upper bound can be attributed to internal coding and, hence, is the result of attentional factors. For a decrement that does not exceed this limit, however, no inference can be made about internal coding or attentional factors. An example of a four-location search versus a two-location 
search, with the boundary line separating the attentional processing (below) from the region where processing does not necessarily involve attentional factors, is shown in Figure 5. In Figures 5 and 8, the coordinates correspond to percent of correct responses instead of percent of decrement; therefore, points falling below the boundary imply attentional, and presumably serial, processing.

Shaw's model has been tested with various kinds of stimuli. It has been shown that no attentional effects occurred in luminance increment detection (Shaw, 1983), sinusoidal grating discrimination (Yager, Kramer, Shaw, \& Graham, 1984), or auditory and visual signal-detection experiments (Mulligan \& Shaw, 1981). For letter-like patterns, however, Shaw's data showed that the decrement in performance exceeded the maximum limit that can be explained by decision processes. Letter detection, therefore, is affected by attentional factors (Shaw, 1983).

In the present experiment, Shaw's model was used to find out (1) if detecting a $\mathrm{T}$ in a field of Ls falls below the boundary line (Figure 5), thus indicating serial processing, and (2) if detecting a + in a field of Ls falls above the boundary. In Shaw's model, points falling above the boundary line do not necessarily indicate parallel processing. But in view of Bergen and Julesz's findings, the assumption of parallel processing would seem to be warranted.

Stimuli. The original software package for texturepattern displays was designed by Peter Burt and later revised and translated into $C$ language by Walter Kropfl. The stimuli were generated on the PDP-11/23 and displayed by the vector system of VRVDP. Each basic element, such as,$+ T$, or $L$, was defined by the user through line drawing instructions at the beginning of the program. Different scale factors could be applied to each basic element. Within each element, a maximum of eight angle rotations in equal steps was allowed. The basic and the transformed individual elements were stored in a linear array. The textured pattern was generated by directing a pointer to each of the desired element, and then displaying that element at a preselected location. A display consisted of four clusters of textured patterns with seven elements in each group. The four patterns were at the vertices of a square, as shown in Figure 6. Trials were grouped in blocks by target element, set size (number of target locations), and eccentricity. Within each block of trials, target and distractor elements remained unchanged.

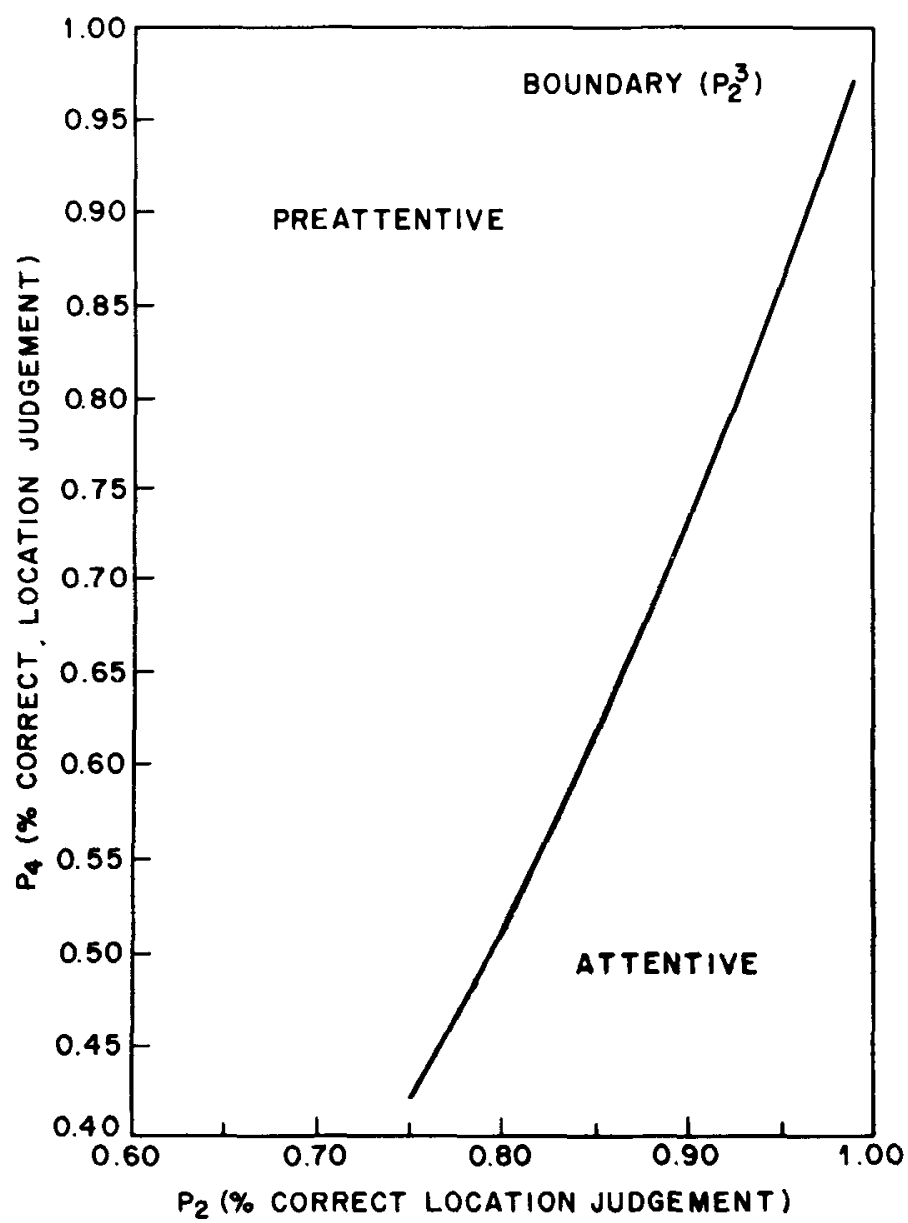

Figure 5. From Shaw's (1980) attention model, boundary line for probability of correct location judgment in the four-tocation tas $\left(P_{4}\right)$ against the probability of correct location judgment in the two-location tast $\left(P_{2}\right)$. Preattentive and attentive labels are used to relate the regions of Julesz's (1984) two visual processes. 


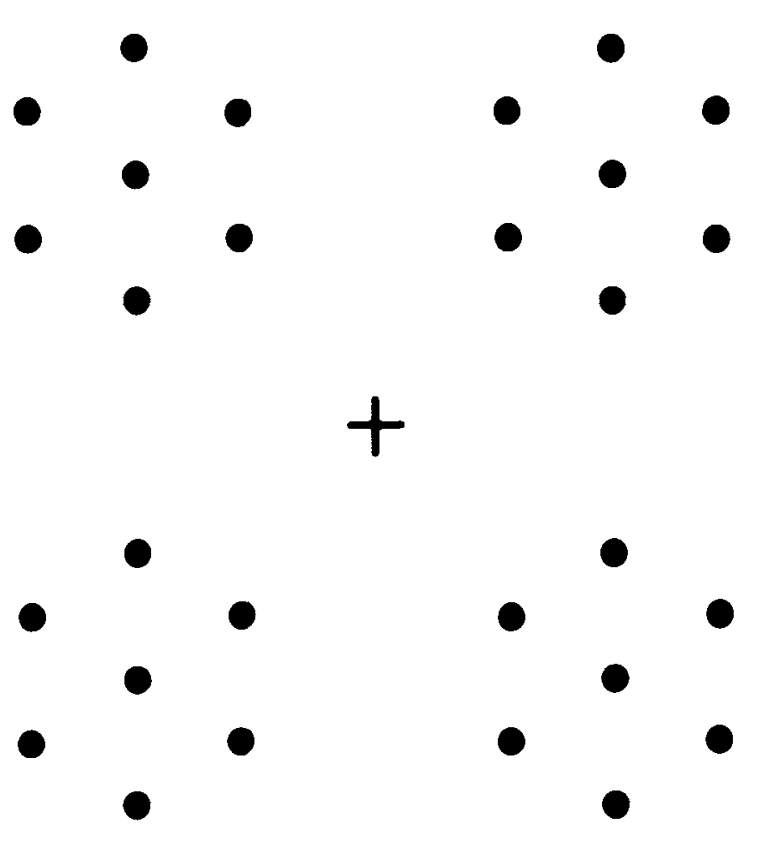

Figure 6. Each dot indicates the location of a feature element. On each trial, four clusters of seven elements in each cluster are displayed. On the two-location trials, two of the displayed clusters are irrelevant. Target element always appears at the center of a cluster.

The distractors were always Ls, irrespective of the target element. Although set size was either two or four, the number of clusters displayed on each trial was always four. This means that when a target could occur only in one of two locations (Set Size 2), two of the displayed clusters were irrelevant. The reason for displaying the same number of clusters was to keep the textured background the same for all tasks. Eccentricity, measured by the distance between diagonal clusters, was $2.9^{\circ}, 3.3^{\circ}$, and $4.0^{\circ}$ of visual angle at a $135-\mathrm{cm}$ viewing distance. Each element subtended 18 min arc. Distance between adjacent elements was about 1.5 times the size of an element. The orientation of each element was chosen at random from eight angles in equal $\left(45^{\circ}\right)$ steps. The target element always appeared in the center of the cluster. The probability that a target appeared in a particular cluster was independent of its appearance in other clusters. Within each cluster, the probability of a target was 0.5 for Set Size 2 and 0.2 for Set Size 4 (Shaw, 1982).

The display was presented at $200 \mathrm{~Hz}$. The luminance of the display was $1.65 \mathrm{fL}$. At the beginning of each trial, there appeared on the scope a fixation point flanked by either two or four dots at the center of those clusters where a target might appear. The observer was instructed to attend to those locations while fixating at the center of the scope. The observer pressed a button to initiate a display when ready. After a 240-msec delay, the stimulus was presented for a brief period, followed by a blank and then by a mask (Figure 7). Masking elements were the union of the target and ground. Observers were required to make two responses: a "yes"-“"no" detection response and a target-localization response. Completion of the responses was followed by a feedback display for $2 \mathrm{sec}$.

Each observer was first tested on the two-location task to determine the SOA ( $\leq 160 \mathrm{msec}$ ) value producing $85 \%-90 \%$ accuracy. The same SOA was then used to obtain the four-location scores. Data were collected from four observers during a period of 7 weeks. The total number of trials for each observer ranged from 12,600 to 19,320 .

Results and discussion. Data were plotted separately for each observer (Figure 8). For observers LB and HG,
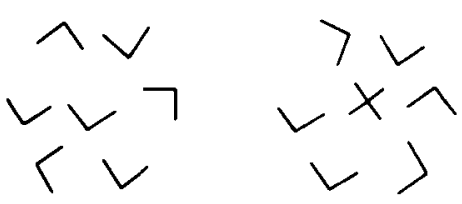

$\bullet$

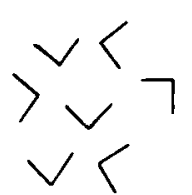

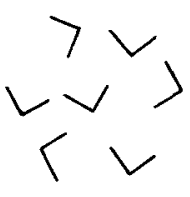
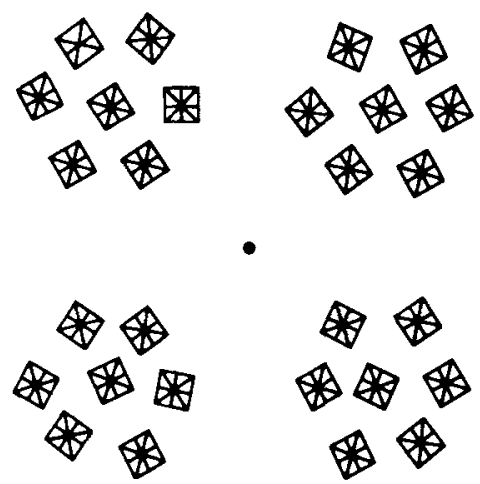

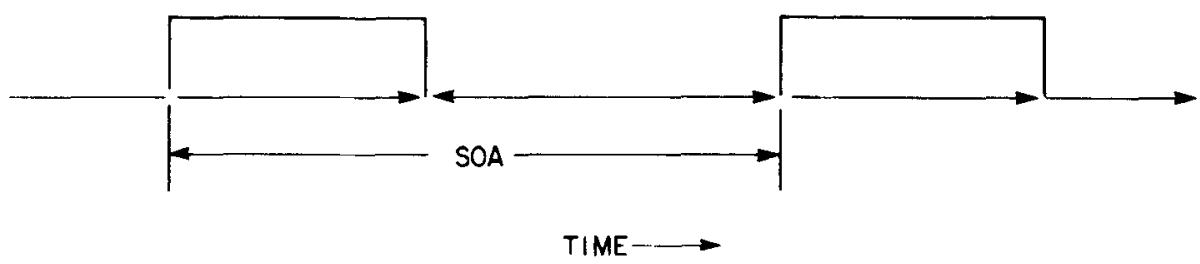

Figure 7. An example of a stimulus presentation. The stimulus (left) consists of seven features at each of the four locations, where $L$ is the distractor and + is the target. On the right is the mask, which is the union of $L, T$, and + . It shows the time course of a trial. Stimulus onset asynchrony (SOA) starts at the onset of the stimulus and lasts through the blank period until the onset of the mask. 


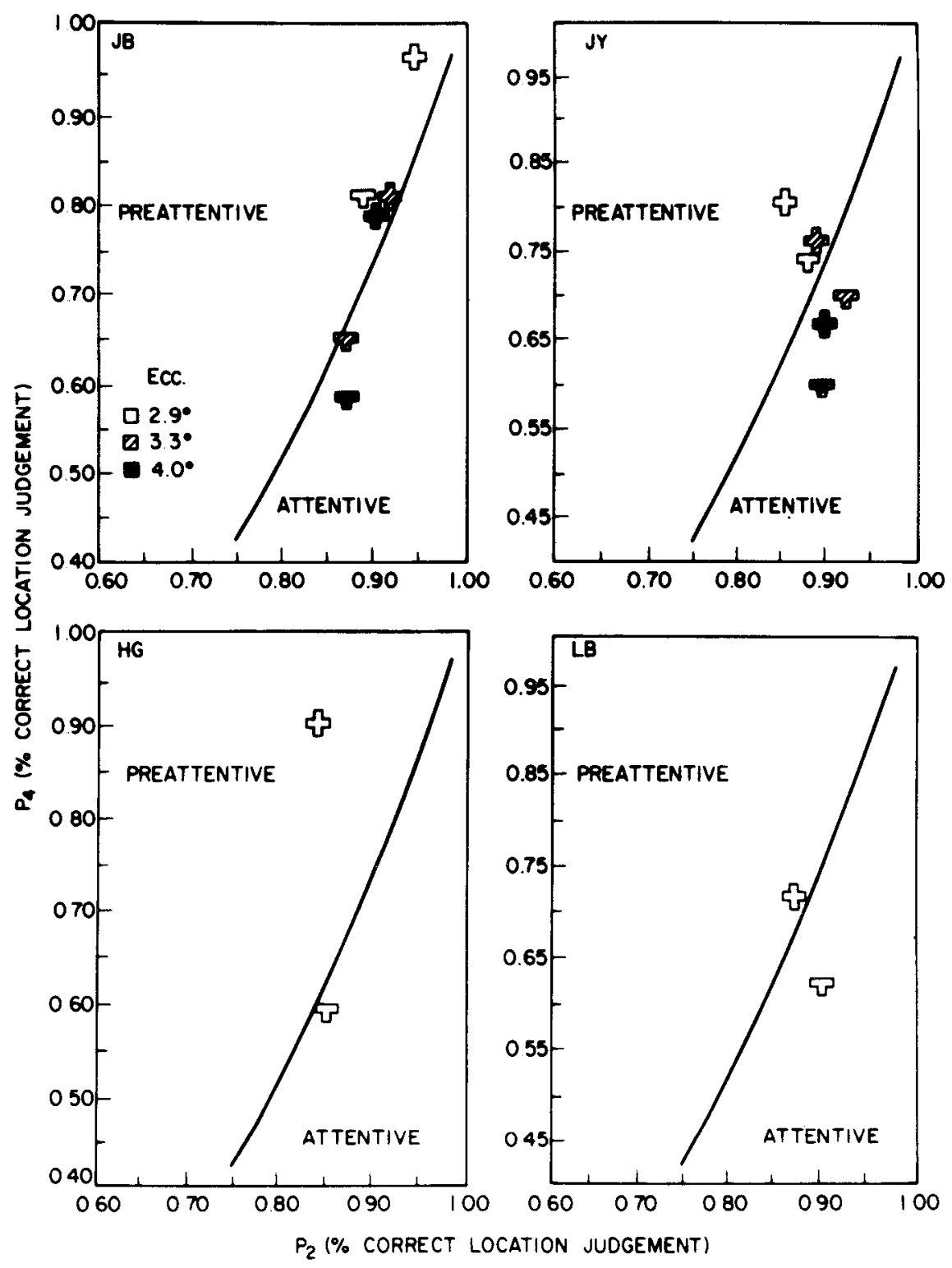

Figure 8. Open symbols, $T$ or + , represent targets presented at $1.45^{\circ}$ from the fixation (or $2.9^{\circ}$ between diagonal clusters); hatched symbols represent targets presented at $1.65^{\circ}$ from the fixation (or $3.3^{\circ}$ in diameter); filled symbols are for targets presented at $2.0^{\circ}$ from the fixation (or $4.0^{\circ}$ in diameter). The boundary line and data points are plotted with probability of correct location judgment in the fourlocation task $\left(P_{4}\right)$ versus the probability of correct location judgment in the two-location task $\left(P_{2}\right)$. SOA ranged from 50 msec for parallel processing of four locations to $90 \mathrm{msec}$ for serial processing of two locations for JB: 90 msec for parallel processing of four locations and $160 \mathrm{msec}$ for serial processing of two locations for LB.

only the smallest $\left(2.9^{\circ}\right)$ eccentricity was used, because they were unable to detect target $\mathrm{T}$ within the allowed SOA of $160 \mathrm{msec}$ at greater eccentricities. The $160-\mathrm{msec}$ limit was used to ensure that no eye movement was allowed. For each eccentricity, a pair of data points representing each target ( $T$ and + ) are plotted in the same shading. Those points that fall above the boundary line indicate parallel processing of either $T$ or + . In other words, with the same available time for processing, performance for four locations was as good as that for two locations. As shown in Figure 8, parallel processing of
+ (above the line) and serial processing of $T$ (below the line) occurred at different eccentricities for different $o b-$ servers. These individual differences among observers could be attributed to practice, since observers JB and JY had been subjects in previous visual search experiments for 18 months and 3 months, respectively, and observers LB and HG had no prior experience. The amount of practice may, in turn, be related to the size of the aperture of attention (Bergen \& Julesz, 1983b; Julesz, 1984) during search in serial as well as in parallel processing (Bergen \& Julesz, 1983b). 
It is important to mention that in the beginning of this study, only the smallest eccentricity was used. Larger eccentricities were introduced when, to my surprise, only two observers (HG and LB) appeared to detect T by serial processing. Unlike Bergen and Julesz's (1983b) study, where the position of the target was randomly assigned among 36 locations, the present study allowed a target to occur in at most four positions. Could it be that for experienced observers, processing $\mathrm{T}$ in Ls did not necessarily involve attentional factors, because the comparison between two and four locations might not be the appropriate test here? Since adding more clusters to the display would be difficult, I decided to move the clusters out into the periphery, expecting that beyond some point parallel processing in certain cases would break down, leaving a clear demarcation between serial and parallel operations. Previous studies have shown that when elements are briefly presented in the foveal region, information is processed in parallel but as elements are moved into the periphery, serial search is required (Beck, 1966; Beck \& Ambler, 1972, 1973). As is clear from the eccentricity data here, the experienced observer can detect $\mathrm{a}+$ in Ls in parallel when elements are as far as $2^{\circ}$ from the fixation point; however, detecting a $\mathrm{T}$ among Ls breaks down around $1.5^{\circ}$. This change in performance with respect to eccentricity could provide information about the aperture of attention. The aperture of attention is defined as the area that can be inspected all at once without moving the eyes. Given a limited search time, a two-location search will produce better performance than will a four-location search, unless the aperture of attention is the same for both two and four locations. Thus, without the involvement of attentional factors, an increase in set size should have no effect on performance; in this case, processing is assumed to be preattentive.

Aperture of attention (AOA) was studied at length by Engel $(1971,1974,1977)$, who found that the size of the conspicuity area (i.e., aperture of attention) was determined by the interaction of eccentric vision and stimulus properties (Engel, 1974). In a more recent study, Bergen and Julesz (1983b) gave a dynamic interpretation of the aperture of attention. They conjectured that in preattentive processing the area of inspection covers a greater area than in attentive processing, where focal attention is required to inspect smaller areas serially. In addition, they postulated a response space where the difference between responses to each stimulus dimension is compared; the finer the positional resolution, the smaller the inspected area in the response space. Julesz (1984) called this region that can be inspected in parallel the aperture of preattentive vision. He explained further in his texton theory that this region can shrink or expand, depending on texton gradient between the target and the distractors. Bergen and Julesz (1983b) also showed that uniform contraction and expansion of stimuli did not affect performance. This implies that the aperture of attention is proportional to positional resolution and element size. The present experiment differed from that of Bergen and Julesz by keeping the size of the elements constant while varying eccentricity. As a result, the AOA must either shrink or enlarge to accommodate the change in texton gradient. In the case of detecting a $\mathrm{T}$ in $\mathrm{Ls}$, increasing the eccentricity decreased the texton gradient, and therefore the AOA was reduced and processing became serial. But for detecting a + in Ls, an increase in eccentricity did not substantially affect the texton gradient, so that information could be processed in parallel within a larger AOA. Further increases in eccentricity, however, would probably show that even $+s$ could not be detected in parallel over the entire visual field. Rentschler and Treutwein (1985), using compound gratings, compared contrast detection to phase discrimination at $0^{\circ}$ and $2^{\circ}$ from the fixation point. Similar to the present findings, their findings showed that performance for contrast (presumably preattentive) and positional discrimination (attentive) differed considerably at $2^{\circ}$ eccentricity, but not at fixation. More recent studies on the distribution of attention at various eccentricities using a cue light either at or some distance from the target have found that a gradient of attention surrounds the target location (Sagi \& Julesz, 1986; Shulman, Wilson, \& Sheehy, 1985). Sagi and Julesz concluded that the aperture of attention scaled with eccentricity with an average diameter of $3^{\circ}$ when the target was presented at $4^{\circ}$ from the fixation, and a diameter of $1.5^{\circ}$ when the target was presented at $2^{\circ}$ from the fixation. In the present experiment, the center of the aperture of attention is always placed at the fixation point, where in the case of Sagi and Julesz's study, the aperture of attention was centered at either $2^{\circ}$ or $4^{\circ}$ from the fixation. If the present results are analyzed in terms of the aperture of attention (AOA), they can be summarized as follows. For observer JB, the maximum AOA is $3.3^{\circ}$ for processing smaller texton gradients (a T in $\mathrm{Ls}$ ) and greater or equal to $4.0^{\circ}$ for processing larger texton gradients ( $a+$ in Ls). For observer JY, the upper limit of AOA for processing a $\mathrm{T}$ in Ls is less than $3.3^{\circ}$ and for $\mathrm{a}+$ in $\mathrm{Ls}$ is between $3.3^{\circ}$ and $4.0^{\circ}$. For observers HG and LB, $2.9^{\circ}$ is the critical AOA separating serial and parallel processing.

In this study, I have presented an estimate of the aperture of attention for processing larger and smaller texton gradients. Aside from the fact that the aperture of attention is a function of texton gradient, set size and eccentricity, it remains to be seen how the aperture of attention varies with texton gradient along other stimulus dimensions, such as luminance, color, or orientation.

\section{Conclusion}

This study, based on Julesz's texton theory and Shaw's attention model, looked at the effects of set size (i.e., the number of target locations), eccentricity, and texton gradient on attention. Bergen and Julesz have shown that detecting a + among Ls is unaffected by set size and is thus mediated by the preattentive system. But detecting a $T$ among $L s$ does depend on set size and requires a serial 
search involving attentive processing. The role of attentional factors in the set size effect was examined by Shaw. She formulated a boundary line that can separate attentive serial processes from those processes that are not necessarily affected by attentional factors. Using Julesz's textons and Shaw's methodology, the present study has shown that whether processing is serial or parallel is a function of eccentricity that differs quantitatively among observers. These differences among subjects can be expressed in terms of the size of the AOA. It was shown that subjects who had participated in similar search tasks for many months were able to inspect larger areas in a shorter time (SOA $=50 \mathrm{msec}$ ) than were less experienced subjects ( $\mathrm{SOA}=90 \mathrm{msec})$. Yet, for each individual, experienced or not, there exist different AOAs for processing various levels of texton gradients. For experienced observers, the upper limit of the AOA for smaller texton gradients (a T in $\mathrm{Ls}$ ) is around $3.3^{\circ}$ in diameter, and for larger texton gradients $(\mathrm{a}+$ in $\mathrm{Ls})$ is up to $4.0^{\circ}$. In the case of inexperienced observers, $2.9^{\circ}$ is the demarcation separating serial from parallel processing.

\section{REFERENCES}

BECK, J. (1966). Perceptual grouping produced by changes in orientation and shape. Science, 154, 538-540.

BECK, J., \& AMBLER, B. (1972). Discriminability of differences in line slope and in line arrangement as a function of mask delay. Perception \& Psychophysics, 12(1A), 33-38.

BeCK, J., \& AMBLER, B. (1973). The effects of concentrated and distributed attention on peripheral acuity. Perception \& Psychophysics, 14, 225-230.

BERGEN, J. R., \& Julesz, B. (1983a). Parallel versus serial processing in rapid pattern discrimination. Nature, 303, 696-698.

BERGEN, J. R., \& Julesz, B. (1983b). Rapid discrimination of visual patterns. IEEE Transactions on Systems, Man, and Cybernetics, SMC$13,857-863$.

BRADDICK, O. (1974). A short range process in apparent motion. Vision Research, 14, 519-528.

BRESENHAM, J. E. (1965). Algorithm for computer control of digital plotter. IBM System Journal, 4, 25-30.

Chang, J. J., \& Julesz, B. (1983). Displacement limits, directional anistropy and direction versus form discrimination in random-dot cinematograms. Vision Research, 23, 639-646.

Chang, J. J., \& Julesz, B. (1984). Cooperative phenomena in apparent movement perception of random-dot cinematograms. Vision Research, 24, 1781-1788.

Chang, J. J., Julesz, B. (1985). Cooperative and non-cooperative processes of apparent movement of random-dot cinematograms. Spatial Vision, 1(1), 39-45.

COOLEY, J. W., \& TUKEY, J. W. (1965). An algorithm for the machine calculation of complex Fourier series. Mathematical Computation, 19, 297-301.

ENGEL, F. L. (1971). Visual conspicuity, directed attention and retinal locus. Vision Research, 11, 563-576.

ENGEL, F. L. (1974). Visual conspicuity and selective background interference in eccentric vision. Vision Research, 14, 459-471.

ENGEL, F. L. (1977). Visual conspicuity, visual search and fixation tendencies of the eye. Vision Research, 17, 95-108.

HuBEL, D. H., \& WIESEL, T. N. (1959). Receptive fields of single neurons in the cat's striate cortex. Journal of Physiology, 148, 574-591.

Hubel, D. H., Wiesel, T. N. (1968). Receptive fields and func- tional architecture of monkey striate cortex. Journal of Physiology, 195, 215-243.

Hubel, D. H., Wiesel, T. N. (1974). Uniformity of monkey striate cortex: A parallel relationship between field size, scatter, and magnification factor. Journal of Comparative Neurology, 158, 295-305.

JuLesz, B. (1960). Binocular depth perception of computer-generated patterns. Bell Systems Technical Joumal, 39, 1125-1162.

JuLesz, B. (1962). Visual pattern discrimination. IRE Transactions on Information Theory, IT-8, 84-92.

JuLESZ, B. (1964). Binocular depth perception without familiarity cues. Science, 145, 356-362.

Julesz, B. (1971). Foundations of cyclopean perception. Chicago: University of Chicago Press.

Julesz, B. (1981). Textons, the elements of texture perception and their interactions. Nature, 290, 91-97.

JULESZ, B. (1984). Toward an axiomatic theory of preattentive vision. In G. M. Edelman, W. E. Gall, \& W. M. Cowan (Eds.) Dynamic aspects of neocortical function (pp. 585-612). New York: Neurosciences Research Foundation, Inc.

Julesz, B., \& ChaNG, J. J. (1976). Interaction between pools of binocular disparity detectors tuned to different disparities. Biological Cybernetics, 22, 107-119.

Julesz, B., \& PAYNe, R. A. (1968). Differences between monocular and binocular stroboscopic movement perception. Vision Research, 8, 433-444.

LaPPIN, J. S., BeLL, H. H. (1976). The detection of coherence in moving random-dot patterns. Vision Research, 16, 161-168.

LoGAN, B. F., JR. (1977). Information in the zero-crossings of bandpass signals. Bell Systems Technical Joumal, 56, 487-510.

Marr, D., \& PoGglo, T. (1976). Cooperative computation of stereo disparity. Science, 194, 283-287.

MARR, D., \& PocGio, T. (1979). A computational theory of human stereo vision. Proceedings of the Royal Society of London, Series B, 204, 301-328.

Mulugan, R., Shaw, M. L. (1981). Attending to simple auditory and visual signals. Perception \& Psychophysics, 30, 447-454.

Nelson, J. J. (1975). Globality and stereoscopic fusion in binocular vision. Journal of Theoretical Biology, 49, 1-88.

Rentschler, I., \& TREUTWEIN, B. (1985). Loss of spatial phase relationships in extrafoveal vision. Nature, 313(24), 308-310.

SAGI, D., JULesz, B. (1986). Enhanced detection in the aperture of focal attention during simple discrimination tasks. Nature, 321, 693-695.

SHAW, M. L. (1980). Identifying attentional and decision making components in information processing. In R. S. Nickerson (Ed.), Attention and performance VIII (pp. 277-296). Hillsdale, NJ: Eribaum.

SHAw, M. L. (1982). Attending to multiple sources of information: 1 . The integration of information in decision making. Cognitive Psychology, 14, 353-409.

SHAW, M. L. (1983). Division of attention among spatial locations: A fundamental difference between detection of letters and detection of luminance increments. In $\mathrm{H}$. Bouma (Ed.), Attention and performance (pp. 109-121). Hillsdale, NJ: Erlbaum.

Shulman, G. L., Wilson, J., \& Sheehy, J. B. (1985). Spatial determinants of distribution of attention. Perception \& Psychophysics, 37, $59-65$.

SperLING, G. (1970). Binocular vision: A physical and a neural theory. The American Joumal of Psychology, 83, 461-534.

Treisman, A., * Gelade, G. (1980). A feature-integration theory of attention. Cognitive Psychology, 12, 97-136.

Tyler, C. W., Julesz, B. (1980). On the depth of the cyclopean retina. Experimental Brain Research, 40, 196-202.

Wilson, H. R., Bergen, J. R. (1979). A four mechanism model for threshold spatial vision. Vision Research, 19, 19-32.

Yager, D., Kramer, P., Shaw, M., \& Graham, N. (1984). Detection and identification of spatial frequency: Models and data. Vision Research, 24, 1021-1035. 